hep-ph/9912504

Published in Phys. Lett. B477, 223 (2000)

\title{
Higgs Mass Prediction with Non-universal Soft Supersymmetry Breaking in MSSM
}

\author{
Sorin Codoban円, Marian Jurčišin 沺 and Dmitri Kazakov [ \\ Bogoliubov Laboratory of Theoretical Physics, JINR , 141980 Dubna, Russia
}

\begin{abstract}
In the framework of the MSSM the non-universal boundary conditions of soft SUSY breaking parameters are considered. Taking as input the top, bottom and Z-boson masses, the values of the gauge couplings at the EW scale and the infrared quasi-fixed points for Yukawa couplings and the soft parameters the mass of the lightest CP-even Higgs boson is found to be $m_{h}=92.7_{-4.9}^{+10} \pm 5 \pm 0.4 \mathrm{GeV} / \mathrm{c}^{2}$ for the low $\tan \beta$ case and $m_{h}=$ $125.7_{-9.0}^{+6.4} \pm 5 \pm 0.4 \mathrm{GeV} / \mathrm{c}^{2}(\mu>0)$ or $m_{h}=125.4_{-9.0}^{+6.6} \pm 5 \pm 0.4 \mathrm{GeV} / \mathrm{c}^{2}(\mu<0)$ in the case of large $\tan \beta$.
\end{abstract}

\section{Introduction}

When making predictions in the framework of the Minimal Supersymmetric Extension of the Standard Model (MSSM), one encounters parameter freedom which is mainly due to the socalled soft SUSY breaking terms. To restrict this freedom and get more predictive power, one usually follows the universality hypothesis that assumes universality (i.e. equality) of soft terms at some high energy scale (most common is the GUT scale at which the gauge couplings are unified). Under this assumption one is left with 5 free parameters, and a thorough analysis of the MSSM mass spectrum in universal case has been done [1].

However, the newest experiments, and first of all recent LEP II limits on the lightest Higgs boson mass [2], suggest that this minimal scenario might not work in practice. In the present paper we try to clarify what one expects to get by releasing the universality conditions in the MSSM, allowing more freedom for the soft terms, with the emphasis on the Higgs boson mass predictions.

In order to see how the non-universality at the GUT scale can change the predictions at the low energy scale, we use recently obtained analytical solutions to the renormalization group (RG) equations for the Yukawa couplings [3, [1]. By means of Grassmannian expansion they

*E-mail: codoban@thsun1.jinr.ru

tE-mail: jurcisin@thsun1.jinr.ru

$\ddagger$ On leave of absence from the Institute of Experimental Physics SAS, Košice, Slovakia

§E-mail: kazakovd@thsun1.jinr.ru 
allow one to derive analytical expressions for soft term evolution [5, 6] and trace analytically the dependence of the soft terms at the $M_{Z}$ scale on their boundary values. In this way, both the expediency of one or another simplifying hypotheses concerning GUT scale values of the soft parameters and the role of non-universality in the context of the MSSM can be estimated.

The parameter space of the MSSM can further be narrowed using the so-called infrared quasi-fixed point (IRQFP) behaviour of some parameters [7], i.e. independence of low energy values on initial conditions at the GUT scale. Using the analytical results along with the numerical ones, one can keep over control the way how the IRQFP strategy works. In what follows we adopt the method advocated in Refs. 8, 9] and apply the above-mentioned tips in making prediction for the lightest Higgs boson mass in the MSSM.

The paper is organized as follows. In Sec. 2, we analyze the IRQFP behaviour of soft parameters with the help of analytical solutions. In Sec. 3 , the low $\tan \beta$ scenario with nonuniversality is investigated, and prediction for Higgs boson mass is given. The same analysis is conducted in Sec. 4 for the case of large $\tan \beta$. Our concluding remarks are presented in Sec. 5 .

\section{Analytical solutions of RG equations for the soft terms and IRQFPs}

As has been recently shown [3], the RGEs for Yukawa couplings can be solved analytically by means of an iteration procedure. Following the recipe advocated in [1], 6], the expressions for soft parameters can be derived from analytical solutions of Yukawa coupling RGEs. Below we provide a brief analysis of analytical solutions for the soft parameters, emphasizing the difference between universal and non-universal cases. For low $\tan \beta$ an exact analytical solution is known [10, 11, 12]; so we discuss just the case of large $\tan \beta$ when all the Yukawa couplings are essential.

Consider solutions to the RG equations for the soft terms. Since the triple scalar couplings $A_{t, b, \tau}$ and gaugino masses $M_{i}$ have a dimension of a mass and the squark, slepton and Higgs mass terms have a dimension of a mass squared, the corresponding RG equations are linear with respect to these parameters, and their solutions can be represented in the form [4

$$
\begin{aligned}
A_{l}(t) & =\sum_{j=t, b, \tau} a_{l j}(t) A_{0 j}+\sum_{k=1,2,3} b_{l k}(t) M_{0 k}, \quad l=t, b, \tau \\
m_{n}^{2}(t) & =\sum_{i, j=1,2,3} c_{i j(n)}(t) M_{0 i} M_{0 j}+\sum_{i, j=t, b, \tau} d_{i j(n)}(t) A_{0 i} A_{0 j}+\sum_{\substack{i=t, b, \tau \\
j=1,2,3}} e_{i j(n)}(t) A_{0 i} M_{0 j}+\sum_{q} k_{q(n)}(t) m_{0 q}^{2}
\end{aligned}
$$

where $m_{n}^{2}$ represent the squark, slepton and Higgs mass terms, $n, q=Q_{3}, U_{3}, D_{3}, H_{1}, H_{2}, E_{3}, L_{3}$ and $m_{0 n}^{2}, A_{0 k}, M_{0 j},(k=t, b, \tau, j=1,2,3)$ are the initial values of the parameters. In one loop order the coefficients of eq.(11) can be calculated within the iteration procedure described in [4]. We have calculated them up to the 6-th iteration, that allows one to get accuracy of $1 \%$. Evaluated at the $M_{Z}$ scale, which corresponds to $t=\log M_{G U T}^{2} / M_{Z}^{2} \approx 66$, they depend on the initial values of Yukawa couplings. In what follows we use the notation $Y_{k} \equiv h_{k}^{2} / 16 \pi^{2}(k=$ $t, b, \tau)$ and $\mathrm{a}_{i} \equiv \alpha_{i} / 4 \pi \equiv g_{i}^{2} / 16 \pi^{2}(i=1,2,3)$. To test the behaviour of the coefficients, we take several sets of Yukawa couplings $Y_{t, b, \tau}^{0}$ at the GUT scale in the range $(0.5 \div 25) \mathrm{a}_{0}$ with some arbitrary ratios of $Y_{b} / Y_{t}$ and $Y_{\tau} / Y_{t}$ ( $\mathrm{a}_{0}$ is the common value of the gauge couplings at the GUT scale). The upper bound for $Y_{i}^{0}$ is taken to preserve the perturbativity up to GUT scale, the lower one keeps us in the large $\tan \beta$ regime. 
In Fig.1 we plot the coefficients of eq.(1) as functions of $Y_{t}^{0} / \mathrm{a}_{0}$. For fixed $Y_{t}^{0}$ different points for a given coefficient correspond to different relative ratios $Y_{t}^{0} / Y_{b, \tau}^{0}$. In this section for illustration we consider three extreme cases: $Y_{t}^{0}=Y_{b}^{0}=Y_{\tau}^{0}, Y_{t}^{0}=Y_{b}^{0}=10 Y_{\tau}^{0}$ and $Y_{b}^{0}=Y_{\tau}^{0}=(1 / 10) Y_{t}^{0}$, to demonstrate relative insignificance of the non-universality of the Yukawa couplings. Indeed, one can see that for $Y_{t}^{0} \geq 2 \mathrm{a}_{0}$ and regardless of the ratios $Y_{t}^{0} / Y_{b, \tau}^{0}$ (but still remaining in the range corresponding to large $\tan \beta$ ) the coefficients of soft breaking parameters in $A_{t, b, \tau}$ approach the asymptotic values equal to their IRQFPs. Since at the same scale one has $M_{1}\left(M_{Z}\right)=0.412 M_{01}, M_{2}\left(M_{Z}\right)=0.822 M_{02}, M_{3}\left(M_{Z}\right)=2.85 M_{03}$, we conclude that the ratios $A_{t, b} / M_{3}$ exhibit the proper IRQFP behaviour as used in Ref. [9].

Hence, non-universality of the soft terms changes almost nothing in the IRQFP behaviour for $A_{t}$ and $A_{b}$ because the coefficient of $M_{03}$ is bigger than the others by a factor of 5 or more. One should also note that non-universality of the Yukawa couplings has a weak effect on the values of the soft terms coefficients. On the plot for $A_{t}$ (the same is true for $A_{b}$ ) for a given coefficient the three points corresponding to various ratios of Yukawa couplings almost overlap. For $A_{\tau}$ all the coefficients but $A_{t}^{0}$ and $M_{01}$ have comparable non-vanishing values and the IRQFP is less stringent. There is also stronger dependence on the relative ratios of Yukawa couplings. Fortunately, $A_{\tau}$ does not play any significant role in the Higgs mass prediction.

The same observations holds true for the soft masses. Taking the values of the Yukawa couplings at the GUT scale as above, we have found that for $m_{Q_{3}}^{2}, m_{U_{3}}^{2}$ and $m_{D_{3}}^{2}$ the prevalence of the gluino mass $M_{03}^{2}$ is obvious and non-universality does not change anything (see on Fig. 1] the $m_{Q_{3}}^{2}$ and $m_{D_{3}}^{2}$ plots) when comparing with the universality case.

In the expression of $m_{H_{1}}^{2}$ the coefficients for some scalar masses are opposite in sign and of the same magnitude $\left(m_{0 H_{1}}^{2}\right.$ comes with '+' sign and $m_{0 Q_{3}}^{2}, m_{0 D_{3}}^{2}$ come with '-' sign), and the same is true for $m_{H_{2}}^{2}$ (with $D \rightarrow U, H_{1} \rightarrow H_{2}$ ). In the case of universal boundary conditions, for the scalar masses $m_{H_{1}}^{2}$ and $m_{H_{2}}^{2}$ the only dependence on the initial conditions left is that on the gluino mass $M_{03}^{2}$ since the scalar mass $m_{0 n}^{2}$ contributions cancel each other. In the case of non-universality with some peculiar choice of initial conditions some residual dependence on the scalar masses may appear. Nevertheless, one can still rely on asymptotic plateau for the coefficients at large Yukawa couplings.

Again, one observes that for $Y_{t}^{0}>2 \mathrm{a}_{0}$ the coefficients approach some asymptotic values and the dependence on non-universality of Yukawa couplings is rather feeble for $m_{Q_{3}}^{2}, m_{U_{3}}^{2}$ and $m_{D_{3}}^{2}$, and small enough for $m_{H_{1}}^{2}$ and $m_{H_{2}}^{2}$. The residual dependence for the coefficients of $M_{03}^{2}$ in the latter case is because we get out of the large Yukawa region: for $Y_{t}^{0}=2 \mathrm{a}_{0}$ we have $Y_{b, \tau}^{0}=0.2 \mathrm{a}_{0}$ which doesn't ensure the IRQFP regime.

The masses of sleptons $m_{E_{3}}^{2}$ and $m_{L_{3}}^{2}$ exhibit rather a fuzzy picture (see the last plot in Fig.(1). The coefficient of $M_{03}^{2}$ is no longer the leading one, instead we have large contributions from $m_{0 E_{3}}^{2}$ and $m_{0 L_{3}}^{2}$. Here some coefficients are negative, thus leading to negative values of e.g. $m_{E_{3}}^{2}$ if $m_{0 L_{3}}^{2}$ and $m_{0 H_{1}}^{2}$ are big enough. The requirement of positiveness of slepton masses imposes some bounds on non-universality choice. In our analysis below we take the relative ratios of the soft masses at the GUT scale in the range $0.5 \div 2$ which ensures $m_{E_{3}}^{2}>0$ for the most regions of the parameter space. These bounds are in agreement with those obtained in [11] in the bottom-up approach. In the universal case both $m_{E_{3}}^{2}$ and $m_{L_{3}}^{2}$ are positive due to the cancellations between different soft terms.

On the plots in Fig.1, only those parameters which have non-negligible coefficients are shown.

Thus, we come to the following conclusions:

i) if the Yukawa couplings at the GUT scale are large enough $\left(>2 \mathrm{a}_{0}\right)$ the coefficients 

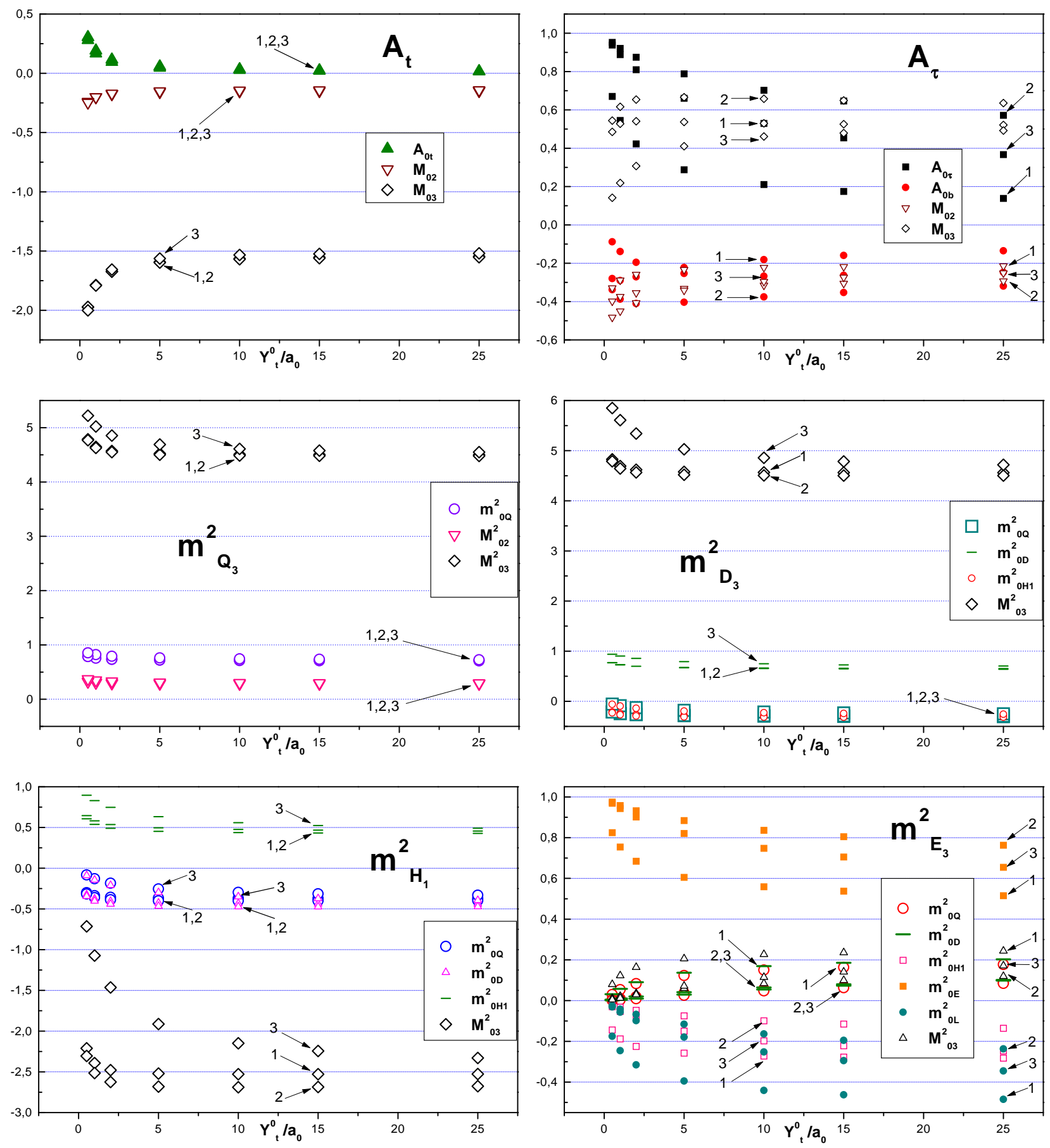

Figure 1: The dependence of the coefficients of eq.(四) on the values of Yukawa couplings. The coefficients are computed at $t=66\left(M_{Z}\right.$ scale). For a given $Y_{t}^{0}$ the plotted points correspond to three different sets of $Y_{b}^{0}, Y_{\tau}^{0}$, namely 1): $\left.\left.Y_{t}^{0}=Y_{b}^{0}=Y_{\tau}^{0}, 2\right): Y_{t}^{0}=Y_{b}^{0}=10 Y_{\tau}^{0}, 3\right)$ : $Y_{b}^{0}=Y_{\tau}^{0}=(1 / 10) Y_{t}^{0}$. Some points for a given $Y_{t}^{0}$ may overlap on the plot. To keep the plots readable no connecting lines between points are drawn, they can be easily recovered due to smooth behaviour. For the same reason the coefficients that are close to zero are not shown. 
of eqs.(1) for the soft terms at low energy scale approach the asymptotic values for both the universal and non-universal boundary conditions, independently of the relative ratios of the Yukawa couplings;

ii) for $A_{t}, A_{b}, m_{Q 3}^{2}, m_{U 3}^{2}, m_{D 3}^{2}, m_{H 1}^{2}$ and $m_{H 2}^{2}$ at the $M_{Z}$ scale the coefficient of $M_{03}\left(M_{03}^{2}\right)$ dominates, the IRQFP behaviour is substantiated and can be used for both the universal and non-universal boundary conditions.

\section{Low $\tan \beta$ Scenario}

We begin our analysis of the influence of non-universality on the mass of the lightest Higgs boson in the low $\tan \beta$ case. The present approach is based on our previous papers [8, 9] where we investigated the mass spectrum of sparticles and the Higgs bosons using the conception of infrared quasi-fixed points (IRQFPs) with the assumption of universality of the soft supersymmetry breaking parameters. The concept of IRQFPs, which has been introduced in [7], was widely employed in the literature [8, 9, 13, 14, 15. It allows one to find the values of the relevant parameters at the $M_{Z}$ scale without exact knowledge of their initial values. The validity of the fixed points is clearly demonstrated in the previous section. This analysis gives us an important information about the weight of various initial parameters in the calculations at low energy values and, finally, in the calculation of the mass spectrum.

In our previous papers [8, 9], we have concluded that all Higgs bosons in the MSSM except the lightest CP-even one, are too heavy to play an important role in the near future experiments; therefore, in the present paper we concentrate on the lightest Higgs boson only.

As input parameters we take the known value of the top-quark pole mass, $m_{t}^{\text {pole }}=173.8 \pm$ $5.2 \mathrm{GeV}$ [16], the experimental values of the gauge couplings [16 $\alpha_{3}=0.120 \pm 0.005, \alpha_{2}=$ $0.034, \alpha_{1}=0.017$ and the sum of the Higgs vev's squared $v^{2}=v_{1}^{2}+v_{2}^{2} \approx 174.1 \mathrm{GeV}^{2}$. We use the approximate and/or numerical solutions of the relevant RG equations to evaluate the fixed point values of the mass parameters. To calculate the mass of the lightest Higgs boson, one also needs to know the ratio $v_{2} / v_{1}$ known as $\tan \beta$ and the mass parameter $\mu$. To determine $\tan \beta$, we use the well-known relation between the top-quark running mass, top Yukawa coupling and $\sin \beta$

$$
m_{t}=h_{t} v \sin \beta \text {. }
$$

The top-quark running mass is found from the pole mass taking into account QCD and SUSY corrections [17, 18 as (for details see Refs. [8, 9])

$$
m_{t}\left(m_{t}\right)=\frac{m_{t}^{\text {pole }}}{1+\left(\frac{\Delta m_{t}}{m_{t}}\right)_{Q C D}+\left(\frac{\Delta m_{t}}{m_{t}}\right)_{S U S Y}} .
$$

The results depend on the sign of the $\mu$ parameter which enters the mixing terms in the stop sector. For $\mu>0$, one obtains $m_{t}\left(m_{t}\right)=162 \pm 5 \mathrm{GeV}$. Negative values of $\mu$ lead to a too light Higgs boson, and we do not consider this case here.

Now we can estimate the value of the $\tan \beta$. We assume that the top Yukawa coupling is close to its IRQFP. This is realized when $\rho_{t 0}=Y_{t}^{0} / \mathrm{a}_{0}>2$. Then one gets $h_{t}\left(M_{Z}\right)=1.09-1.14$ when $2<\rho_{t 0}<25$. As a central value of top Yukawa coupling we take $h_{t}\left(M_{Z}\right)=1.12$ which corresponds to $\rho_{t 0}=5$. This gives the following value of $\tan \beta$ :

$$
\tan \beta=1.47 \pm 0.1 \pm 0.15 \pm 0.05, \quad \mu>0 .
$$


The deviations from the central value are connected with the deviation from the fixed point value of the Yukawa coupling and the experimental uncertainties in the top-quark mass and $\alpha_{3}\left(M_{Z}\right)$, respectively.

The Higgs mixing parameter $\mu$ can be determined from the requirement of radiative EWSB and can be found from the Higgs potential minimization condition. In contrast with our previous paper [8] (where we have taken into account only tree level minimization condition), we include here the one-loop corrections. It gives the difference of about $2 \mathrm{GeV}$ for the lightest Higgs boson mass. The one-loop minimization condition reads

$$
\frac{M_{Z}^{2}}{2}+\mu^{2}=\frac{m_{H_{1}}^{2}+\Sigma_{1}-\left(m_{H_{2}}^{2}+\Sigma_{2}\right) \tan ^{2} \beta}{\tan ^{2} \beta-1}
$$

where $\Sigma_{1}$ and $\Sigma_{2}$ are the one-loop corrections [19], $M_{Z}$ is the $Z$-boson mass and $m_{H_{1}}^{2}$ and $m_{H_{2}}^{2}$ are the Higgs soft mass parameters which are determined by solutions of the RG equations. The latter possess the IRQFP's which we use in our analysis. The above equation allows one to obtain an absolute value of $\mu$. The sign of $\mu$ remains a free parameter, however, as it has already been mentioned, negative values of $\mu$ give too small values of the lightest Higgs boson mass and are excluded experimentally [2].

In the MSSM, the Higgs sector consists of five physical states: two neutral CP-even scalars $h$ and $H$, one neutral CP-odd scalar $A$, and two charged Higgs scalars $H^{ \pm}$. We concentrate on the mass of the lightest Higgs boson $h$. At the tree level, the mass of $h$ is smaller than the mass of $Z$-boson, $M_{Z}$, but the loop corrections increase it. In general, the mass matrix for the CP-even neutral Higgs bosons looks like

$$
\mathcal{M}=\left(\begin{array}{cc}
\tan \beta & -1 \\
-1 & \cot \beta
\end{array}\right) m_{A}^{2} \cos \beta \sin \beta+\left(\begin{array}{cc}
\cot \beta & -1 \\
-1 & \tan \beta
\end{array}\right) M_{Z}^{2} \cos \beta \sin \beta+\left(\begin{array}{ll}
\Delta_{11} & \Delta_{12} \\
\Delta_{12} & \Delta_{22}
\end{array}\right)
$$

where $m_{A}$ is the mass of the CP-odd Higgs boson and $\Delta^{\prime} s$ are the radiative corrections 20]. These corrections depend on stop masses which are given by the following equation:

$$
\tilde{m}_{t_{1,2}}^{2}=\frac{1}{2}\left[\tilde{m}_{t_{L}}^{2}+\tilde{m}_{t_{R}}^{2} \mp \sqrt{\left(\tilde{m}_{t_{L}}^{2}-\tilde{m}_{t_{R}}^{2}\right)^{2}+4 m_{t}^{2}\left(A_{t}-\mu \cot \beta\right)^{2}}\right],
$$

To find the Higgs boson mass, one has to diagonalize the mass matrix (5). In our previous paper [8] we have estimated the mass of the lightest Higgs boson exploring the IRQFPs in case of universal boundary conditions. In the present paper, we also use the concept of IRQFPs but release the universality conditions and allow moderate deviations from universality. In view of the analysis of the previous section, we expect that the main influence of non-universality comes from the initial values of the Higgs masses $m_{H_{1}}^{2}$ and $m_{H_{2}}^{2}$ and that of $m_{U}^{2}$ while those of $m_{Q}^{2}$ and gauginos are of minor importance. In the numerical analysis, we consider the following intervals for the top Yukawa coupling and soft breaking parameters at the GUT scale: $Y_{t}^{0} / \mathrm{a}_{0} \in<2,25>, A_{0 t} / M_{03} \in<-1,1>, m_{0 i}^{2} / M_{03}^{2} \in<0.25,4>$ and $M_{0 j} / M_{03} \in<0.5,2>$, where $i=\left(Q_{3}, U_{3}, H_{1}, H_{2}\right)$ and $j=1,2$.

In Fig. 2, the dependence of the mass of the lightest Higgs boson $m_{h}$ on the geometric mean of stop masses $\sqrt{\tilde{m}_{t_{1}} \tilde{m}_{t_{2}}}$ is shown, which is often identified with the supersymmetry breaking scale $M_{S U S Y}$. One can see obvious saturation of the Higgs mass when $M_{S U S Y} \geq 500 \mathrm{GeV}$. The central (dash) line corresponds to the central values of the parameters. We take them as follows: $Y_{t}^{0} / \mathrm{a}_{0}=5, A_{0 t} / M_{03}=0, m_{0}^{2} / M_{03}^{2}=1$ for all scalar masses and $M_{0 j} / M_{03}=1$ for 

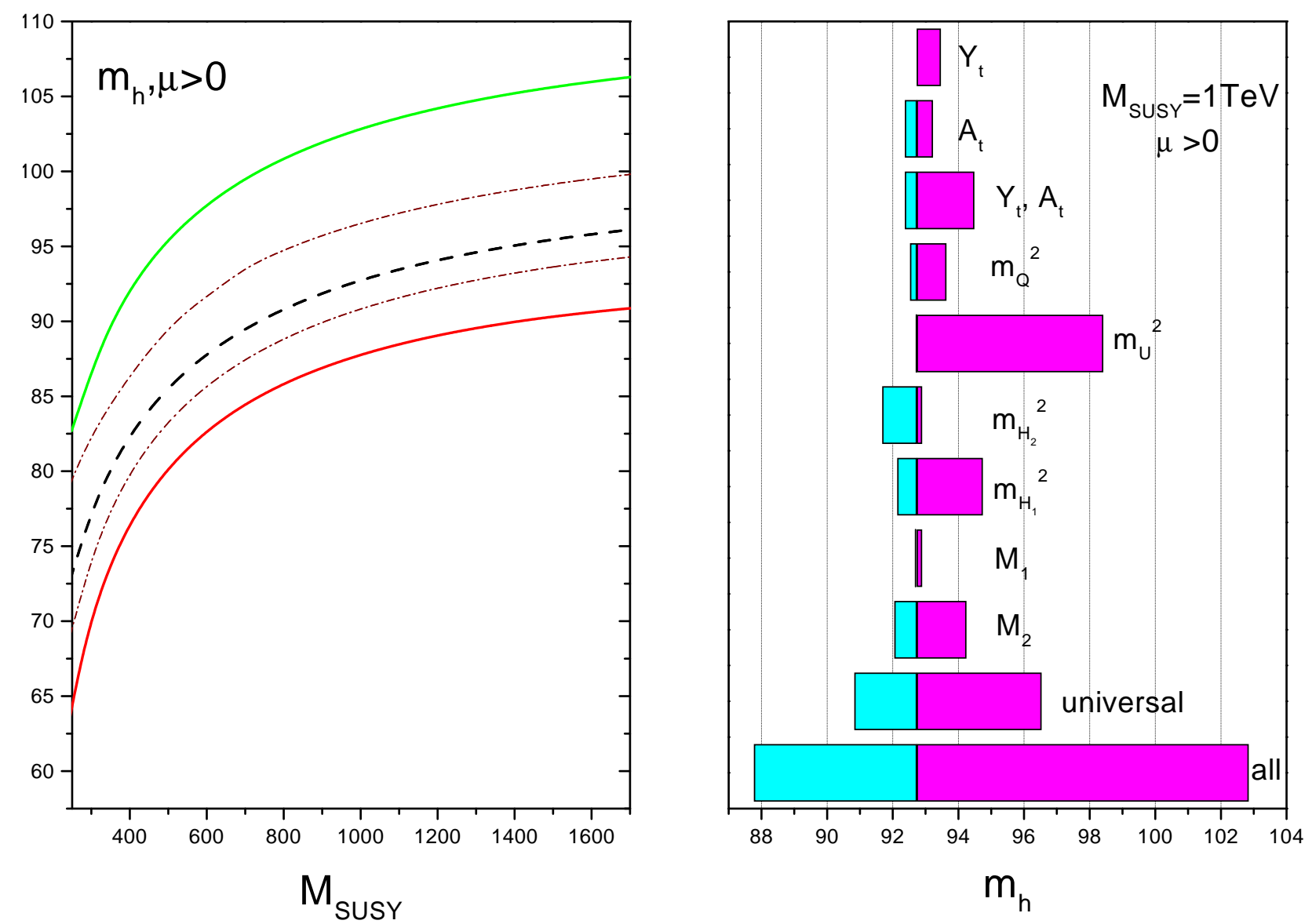

Figure 2: The mass of the lightest Higgs boson as a function of $M_{S U S Y}$. The dashed (central) line corresponds to the central values of the parameters, the dash-doted lines corresponds to the upper and lower limits in the case of universal boundary conditions and the solid lines are absolute upper and lower limits on the mass of the lightest Higgs boson in the non-universal case (left). The influence of variations from central values of the individual parameters as well as their collective effect on the mass of the lightest Higgs boson in both the universal and non-universal cases at a typical scale $M_{S U S Y}=1 \mathrm{TeV}$ (right).

gaugino masses $M_{01}$ and $M_{02}$. If one assumes the universality, the mass of the lightest Higgs boson at a typical scale $M_{S U S Y}=1 \mathrm{TeV}(\mu>0)$ is

$$
m_{h}=92.7_{-1.9}^{+3.8} \pm 5 \pm 0.4 \mathrm{GeV}, \quad \text { for } M_{S U S Y}=1 \mathrm{TeV} .
$$

The first uncertainty is given by the deviations from central values of the top Yukawa coupling and soft breaking parameters $(+3.8(-1.9))$, the second one by the uncertainty of top-quark mass and the third one by uncertainty in the strong coupling constant $\alpha_{3}\left(M_{Z}\right)=0.120 \pm 0.005$. If the parameters are non-universal at the GUT scale, the range of possible values of the lightest Higgs boson mass becomes wider:

$$
m_{h}=92.7_{-4.9}^{+10.1} \pm 5 \pm 0.4 \mathrm{GeV}, \quad \text { for } M_{S U S Y}=1 \mathrm{TeV} .
$$

From Fig.2, one can see that the main deviations from the universal case for the lightest Higgs boson mass is due to the soft mass parameters, especially $m_{U}^{2}$. One can see that the restrictions 

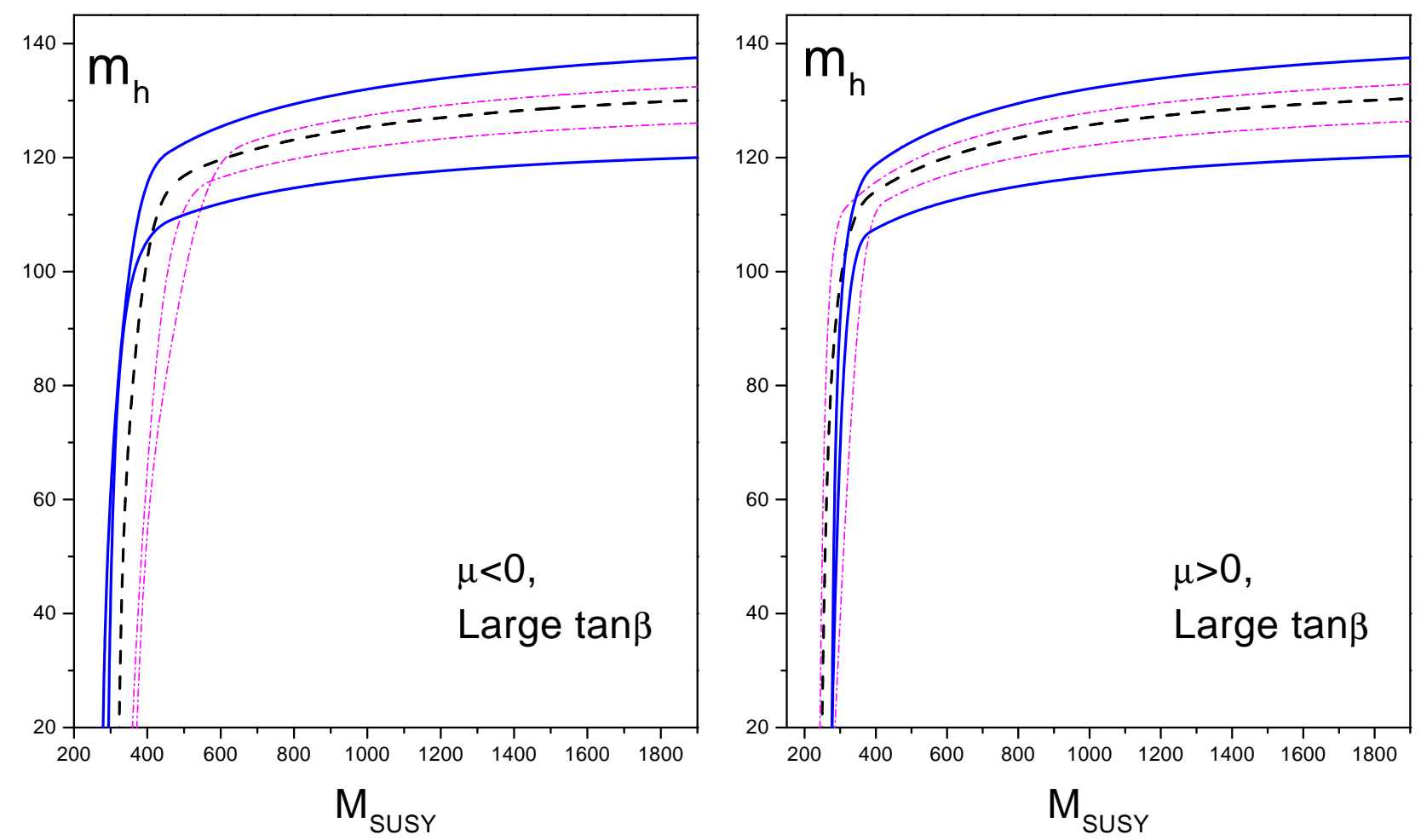

Figure 3: The mass of the lightest Higgs boson as a function of $M_{S U S Y}$ for both the cases $\mu>0$ and $\mu<0$. The dashed (central) line corresponds to the central values of the parameters, the dash-doted lines correspond to the upper and lower limits for the universal boundary conditions and the solid lines are the absolute upper and lower limits on the mass of the lightest Higgs boson in the non-universal case. The line intersection is related to a steep fall of $m_{h}^{2}$ at low values of $M_{S U S Y}$ where $m_{h}^{2}$ becomes negative. The position of the "switch" depends on the choice of parameters. Physically relevant parts of the plots start at approximately $M_{S U S Y} \geq 400$ $\mathrm{GeV}(\mu>0)$ and $M_{S U S Y} \geq 600 \mathrm{GeV}(\mu<0)$

on the lightest Higgs boson mass in the non-universal case are not so strict as in the universal one. In the non-universal case, in the MSSM with low $\tan \beta$ it is possible that the lightest Higgs boson has the mass slightly higher than $100 \mathrm{GeV}$ contrary to the universal case. However, it is still too light in view of recent experimental data, which sets the lower limit on the Higgs mass of $103 \mathrm{GeV}$ [2].

Of course, if one allows the parameters to have larger deviations than we use in present analysis (we have imposed the following restrictions on the soft masses: $m_{0 i}^{2} / M_{03}^{2} \in<.25,4>$ and $m_{0 i}^{2} / m_{0 j}^{2} \in<1 / 16,16>$ where $\left.i, j=Q_{3}, U_{3}, D_{3}, H_{1}, H_{2}\right)$ it is possible to find the mass of the lightest Higgs boson even larger than our upper bound. For instance if one allows the soft masses to be in interval $m_{0 i}^{2} / m_{0 j}^{2} \in<1 / 100,100>$ than the upper bound for $m_{h}$ increases by about $3 \mathrm{GeV}$. However, we consider such a large non-universality to be unnatural. 


\section{$4 \quad$ Large $\tan \beta$ scenario}

Consider now the large $\tan \beta$ case. The situation is more complicated because the space of input parameters is larger. We follow the same strategy as in Ref. 9], but with some modifications. To take into account the non-universality, we keep the initial values of top and bottom Yukawa couplings within the whole interval $\rho_{t 0}, \rho_{b 0} \in<2,25>$ (see the previous section for notation). In Ref. [9], we restricted this interval imposing some constraints on the $\sin \beta$ and $\tau$-lepton mass $m_{\tau}$. Here, the only restriction is the attraction to the IRQFPs which defines the above mentioned interval. To determine $\tan \beta$, we use equation (2) and a similar one for the bottom-quark running mass

$$
m_{b}=h_{b} v \cos \beta
$$

so that $\tan \beta$ is defined from

$$
\tan \beta=\frac{m_{t}}{m_{b}} \frac{h_{b}}{h_{t}} .
$$

The top-quark running mass has been calculated in the previous section. As for the bottomquark running mass, the situation is more complicated because the mass of the bottom $m_{b}$ is essentially smaller than the scale $M_{Z}$; so we have to take into account the running of this mass

from $m_{b}$ to the $M_{Z}$ scale. The bottom-quark pole mass is $m_{b}^{\text {pole }}=4.94 \pm 0.15 \mathrm{GeV}$ [21]. To calculate the running mass, we use the well-known procedure given e.g. in Refs. [9, 18, 22, 23]. Since we assume non-universality of the Yukawa couplings, the value of $\tan \beta$ obtained from eq.(10) belongs to a wider interval. For the central values of the Yukawa couplings and the mass parameters (see the previous section) we find the following values of $\tan \beta$ : $\tan \beta=69.3, \mu>0$ and $\tan \beta=38.1, \mu<0$. When the parameters vary around their central values, $\tan \beta$ is changing within the intervals:

$$
\tan \beta \in<41.2,130.2>\quad(\mu>0), \quad \tan \beta \in<35.0,40.6>\quad(\mu<0) .
$$

The parameter $\mu$ is calculated in the same manner as in the low $\tan \beta$ case. The same is true for the stop and sbottom masses. In Fig. 3 , we present the dependence of the mass of the lightest Higgs boson on $M_{S U S Y}$ for both the cases $\mu>0$ and $\mu<0$. One can immediately see the very sharp increase of $m_{h}$ to the plateau starting from $M_{S U S Y} \geq 500 \mathrm{GeV}$. In the non-universal case, the interval of masses is slightly wider than in the universal one. Fig. 1 shows the dependence of the lightest Higgs boson mass on the deviations of the individual parameters from their central values for both the cases $\mu>0$ and $\mu<0$. The major influence on the Higgs mass is given by the Yukawa top and bottom couplings. The influence of the other parameters is negligible. One can immediately see the big difference between the universal and non-universal cases. If one assumes the universality of the Yukawa couplings and soft parameters, the mass of the lightest Higgs boson at the typical scale $M_{S U S Y}=1 \mathrm{TeV}$ is given by

$$
\begin{aligned}
& m_{h}=125.7_{-3.5}^{+2.2} \pm 5 \pm 0.4 \mathrm{GeV} \text { for } \mu>0 \\
& m_{h}=125.4+2.0 \pm 5 \pm 0.4 \mathrm{GeV} \text { for } \mu<0 .
\end{aligned}
$$

The first uncertainty is connected with the deviations of the Yukawa couplings and soft parameters from their central values in the universal case, the second one is due to the experimental uncertainty in the top-quark mass, and the third one is connected to that of the strong coupling constant. When one does not assume universality, the allowed interval of the Higgs boson mass 

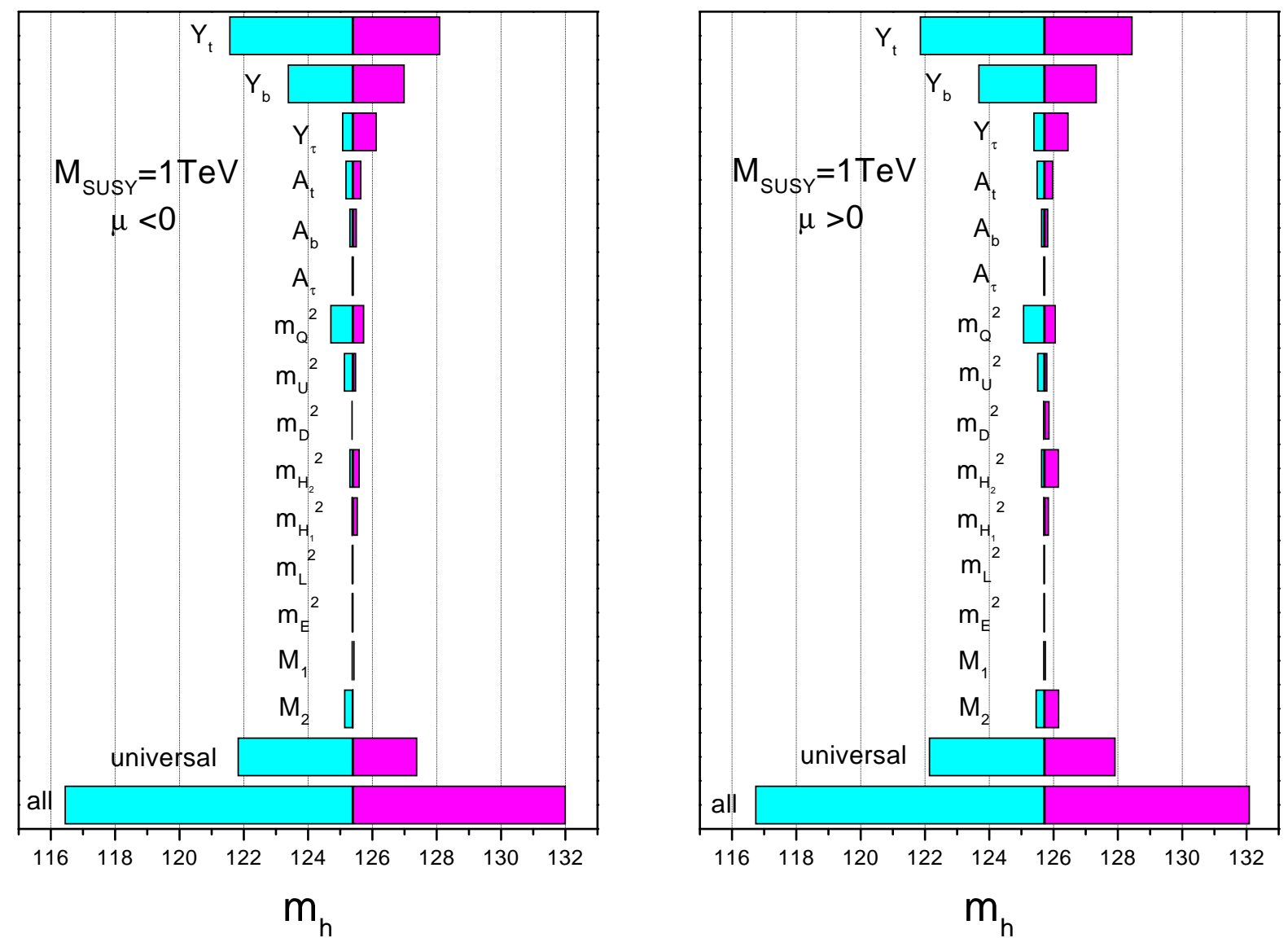

Figure 4: The influence of the variations from central values of the individual parameters and their collective effect on the mass of the lightest Higgs boson in both the universal and non-universal cases at a typical scale $M_{S U S Y}=1 \mathrm{TeV}$.

is wider. For $M_{S U S Y}=1 \mathrm{TeV}$ we get

$$
\begin{aligned}
& m_{h}=125.7_{-9.0}^{+6.4} \pm 5 \pm 0.4 \mathrm{GeV} \text { for } \mu>0 \\
& m_{h}=125.4+6.6 \pm 5 \pm 0.4 \mathrm{GeV} \text { for } \mu<0 .
\end{aligned}
$$

One can see that in the case of large $\tan \beta$ the mass of the lightest Higgs boson typically belongs to the interval $<115,130>\mathrm{GeV}$. The upper bound on $m_{h}$ is reached for $Y_{t}^{0}$ close to its perturbative limit $\left(Y_{t}^{0} / \mathrm{a}_{0} \approx 25\right)$. The influence of the soft parameters is small as one can see in Fig. 1 and is also restricted by the assumption that the soft masses for sleptons $m_{E 3}^{2}$ and $m_{L 3}^{2}$ are positive at $M_{Z}$ scale. The lower bound on $m_{h}$ decreases by about $3 \mathrm{GeV}$ when the assumption of IRQFP for Yukawa couplings is released. There is still a constraint on $Y_{t}^{0}$ $\left(Y_{t}^{0} / \mathrm{a}_{0}>1.2\right)$ given by condition $\sin \beta \leq 1$ in the relation for top mass (2). Experiments are still far away from these values, though the lower boundary may be within the reach of LEP II. 


\section{Conclusion}

We have analyzed the influence of non-universality of the Yukawa couplings and soft SUSY breaking parameters on the mass of the lightest Higgs boson $h$ in the MSSM. Possible values of the Higgs mass are obtained. This may be important for the Higgs searches in the nearest future.

In the low $\tan \beta$ case, the main role is played by non-universality of the mass soft parameters. Assuming a moderate deviation from universality one gets the mass of the lightest Higgs boson below $103 \mathrm{GeV}$ which is almost excluded by recent experimental data [2].

For high $\tan \beta$ the situation is different. Here, the main role is played by non-universality of the Yukawa couplings; the variations of the soft terms are of minor importance. The mass of the lightest Higgs boson in this case is much larger. Here more interesting is the lower bound of the Higgs mass. The effect of non-universality is the decrease in this bound which may become as low as $115 \mathrm{GeV}$ leaving hopes for the imminent observation of the Higgs boson.

\section{Acknowledgments}

We are grateful to G. Moultaka for useful discussions. Financial support from RFBR grants \# 99-02-16650 and \# 96-15-96030 is kindly acknowledged.

\section{References}

[1] G. G. Ross, R. G. Roberts Nucl. Phys. B377 (1992) 571

W. Barger, M. Berger, P. Ohman, Phys. Rev. D49 (1994) 4908

M. Carena, C. E. M. Wagner, Nucl. Phys. B452 (1995) 45

W. de Boer, R. Ehret, D. I. Kazakov, Z. Phys. C67 (1995) 647

W. de Boer, R. Ehret, D. I. Kazakov, Z. Phys. C71 (1996) 415.

[2] The Higgs Working Group and the LEP experiments, "LEP Higgs Working Group Status Report", CERN-LEPC 99-6, Sept. 9, 1999.

[3] G. Auberson, G. Moultaka, Eur. Phys. J. C12 (2000), 331-342 hep-ph/9907204,

[4] D. Kazakov, G. Moultaka, hep-ph/9912271, to appear in Nucl. Phys. B

[5] L.A.Avdeev, D.I.Kazakov and I.N.Kondrashuk, Nucl. Phys. B510 (1998) 289, hepph/9709397

[6] D.I.Kazakov, Phys. Lett. B449 (1999) 201, hep-ph/9812513.

[7] C. T. Hill, Phys. Rev. D24 (1981) 691; C. T. Hill, C. N. Leung, and S. Rao, Nucl. Phys. B262 (1985) 517.

[8] G. K. Yeghiyan, M. Jurčišin, D. I. Kazakov Mod. Phys. Lett. A14 (1999) 601, hepph/9807411

[9] M. Jurčišin, D. I. Kazakov Mod. Phys. Lett. A14 (1999) 671, hep-ph/9902290

[10] L.E.Ibáñez, C. López and C.Muñoz, Nucl. Phys. B256 (1985) 218. 
[11] M. Carena, P. Chankowski, M. Olechowski, S. Pokorski and C.E.M. Wagner Nucl. Phys. B491 (1997) 103, hep-ph/9612261.

[12] S. Codoban, D.I. Kazakov, hep-ph/9906256, to appear in Eur. Phys. J. C

[13] M. Carena, M. Olechowski, S. Pokorski, C. E. M. Wagner, Nucl. Phys. B419 (1994) 213.

[14] M. Carena, C. E. M. Wagner, Nucl. Phys. B452 (1995) 45,

M. Lanzagorta, G. Ross, Phys. Lett. B364 (1995) 163,

J. Casas, J. Espinosa, H. Haber, Nucl. Phys. B526 (1998) 3.

[15] W. Bardeen et al, Phys. Lett. B320 (1994) 110.

[16] Review of Particle Properties, Eur. Phys. J. C3 (1998)

[17] B. Schrempp, M. Wimmer, Prog. Part. Nucl. Phys. 37 (1996) 1.

[18] J.A. Bagger, K. Matchev and D.M. Pierce, Phys. Lett. B348 (1995) 443,

D. M. Pierce, J. A. Bagger, K. Matchev and R. Zhang, Nucl. Phys. B491 (1997) 3.

[19] A.V. Gladyshev, D.I. Kazakov, W. de Boer, G. Burkart, R. Ehret, Nucl. Phys. B498 (1997) 3 .

[20] J. Ellis, G. L. Fogli, E. Lisi, Phys. Lett. B333 (1994) 118,

R. Hempfling, A. Hoang, Phys. Lett. B331 (1994) 99,

R. Hempfling, Phys. Rev. D49 (1994) 6168,

M. Carena, J. R. Espinosa, M. Quiros, C. E. M. Wagner, Phys. Lett. B355 (1995) 209,

M. Carena, M. Quiros, C.E.M. Wagner, Nucl. Phys. B461 (1996) 407,

S. Heinemeyer, W. Hollik, G. Weiglein, Phys. Lett. B455 (1999) 179,

S. Heinemeyer, W. Hollik, G. Weiglein, Eur. Phys. J. C9 (1999) 343.

[21] C. T. H. Davies, et al., Phys. Rev. D50 (1994) 6963.

[22] H.Arason, D. Castano, B. Keszthelyi, S. Mikaelian, E, Piard, P. Ramond, and B. Wright, Phys. Rev. D46 (1992) 3945.

[23] N.Gray, D. J. Broadhurst, W. Grafe, and K. Schilcher, Z. Phys. C48 (1990) 673. 